\title{
Visual Research Methods: Qualifying and Quantifying the Visual
}

\author{
Patricia A. L. ONG \\ School of Education, University of Waikato, New Zealand \\ pong@waikato.ac.nz; patricia.alpong@gmail.com
}

\begin{abstract}
The role of visual research methods in ethnographic research has been significant, particularly in place-making and representing visual culture and environments in ways that are not easily substituted by text. Digital media has extended into mundane, everyday existences and routines through most noticeably the modern smartphone, social media and digital artefacts that have created new forms of ethnographic enquiry. Ethnographers have engaged in this relatively new possibility of exploring how social media and new technologies transform the way we view social realities through the digital experience. The paper discusses the possible role of visual research methods in multimethod research and the theoretical underpinning of interpreting visual data. In the process of interpreting and analysing visual data, there is a need to acknowledge the possible ambiguity and polysemic quality of visual representation. It presents selectively the use of visual methods in an ethnographic exploration of early childhood settings through the use of internet-based visual data, researcher and participantgenerated visual materials and media, together with visual-elicited (e.g. drawings, still images, video clips) information data through several examples. This approach in 'visualizing' the curriculum also unveils some aspects of the visual culture or the 'hidden curriculum' in the learning environment.
\end{abstract}

\section{Keywords}

visual research - visual ethnography - polysemic representation 
Although visual methods have become increasing importance, it has traditionally taken a secondary place when compared to narrative approaches based on text and verbal discourse. The internet and electronic communications have made an attentiveness to the 'visual' essential in education and educational research. Qualitative researchers have made progress in developing visual methodologies to study visual culture and phenomena (Metcalfe, 2016; Prosser, 2007). The issue of new technologies and developments producing shifts in the way we conceptualize and experience social and electronic realities that we experience (Sarah Pink, 2012). Ethnographers have the option to explore the ways in which these new technologies, software and images have become part of their social reality and that their focus may be on how these technologies are appropriated rather than how they transform the basis of the world that we live in (Coleman, 2010; Miller, 2011). The role of visual methodologies and ethnography in looking at how the curriculum is enacted and articulated in everyday practice will be explored.

Visual ethnographic study explores the complex interactions and relationships between local practices of the study and global implications and influences of digital media, the materiality and the politics of representation. The representation through visuality of digital media includes the mundane, everyday routines, the manifestation of cultural life and modes of communication. Media in many instances have become central to the articulation and expression of valued beliefs, ceremonious practices and modes of being (Coleman, 2010). It is therefore essential to press beyond the boundaries of narrow presumptions about the limitations of the digital experience.

Visual ethnography engages with methods through its process of research, analysis and representation. It is inescapably collaborative, to a certain extent is participatory, involves analysing visual cultures, and requires an understanding of how the data set materials from both researcher and participant relate to one another. The process of audio-visual recording of research participants while 'walking with them' produces a research encounter that captures the 'place in a phenomenological sense (Pink, 2014). These processes constitute multisensory experiences and a collaborative work of visual (audio) ethnographic representations of urban contexts in the case study. Visual ethnography through photography and video captures a sense of a place, its history and cultural contexts, maybe everyday life, routines, languages, social interactions and gestures of communication, with other material and sensorial realities of the environment and place.

The gathering of pre-existing societal imagery and found imagery although usually regarded as secondary data requires a minimum reflexive knowledge 
of the technical and expressive aspects of imagery and representational techniques so as to be able to read and utilize them in an appropriate way. Therefore, some form of visual competence is required and the audience often pays attention to the historical and cultural aspects and contexts of production and consumption (Pauwels, 2007). Researcher-generated imagery requires a sufficient degree of technical expertise that allow them to produce images and other forms of visual representations and that they are aware of cultural conventions and perceptual principles of the academic or non-academic audience that they aim to address. Visual ethnography is also concerned with understanding how we know as well as the environments in which knowledge is generated and it involves engaging with the philosophy of knowledge, of practice and of the place and space (Sarah Pink, 2014). This form of methodological focus through the visual requires a commitment to visual theory and researcher positionality particularly with respect to the literal and figurative aspects of one's perspective (Metcalfe, 2016).

Visual culture becomes ingrained in the school culture that is typically unquestioned and unconscious, but it forms a 'hidden curriculum' because it is both visual yet unseen. The organizational culture is influential in the organization's outcomes as the 'ethos' links it with the school culture and ultimately the organization's effectiveness. The organizational culture through ethnographic methodological framework allows an analytic approach to understanding the processes and rationale behind 'school life' (Prosser, 2007). The debate goes on regarding the significance of the visual culture of schools and centres and the argument that visual culture and image-based methodologies are as important as number and word-based methodologies in the constructions of school culture and its influence on education policy. Visual-centric approach highlights and gives priority to what is visually perceived rather than what is written, spoken or statistically measured. Observed events, routines, rituals, artefacts, materials, spaces and behaviours in everyday routines are the evidence and markings of the past, present and future hidden curriculum.

The following sections discuss the methodological, theoretical and conceptual frameworks through which visual data may be interpreted. A combination of methodological strategies, empirical approaches, perspectives and interpretive-analytic stances enhances the rigor, depth and complexity of the research inquiry (Denzin, 2012; Flick, 2018).

The nature of visual research methods has posed some challenges based on issues of concern regarding the validity and rigor of such approaches. This has 
led to some challenges in identifying studies that integrate these methods with mixed methods research that use both quantitative and qualitative strategies (Shannon-Baker \& Edwards, 2018). The intersection of visual methods with mixed methods research allow complements and expansion of qualitative and quantitative data and the approach is also in alignment with philosophical and theoretical assumptions (Clark \& Ivankova, 2016), ShannonBaker \& Edwards, (2018) points out that there are methodological differences between a mixed methods study that utilizes visual research methods and visual methods study that utilizes mixed methods approaches. Studies using visual methods are often paired with qualitative methods such as interviewing and written reflective logs and the use of multiple methods speak to diverse experiences and contribute to the philosophical belief in multiple truths (O'Connell, 2013; Prosser, 2007; Rule \& Harrell, 2010). The challenges in using visual methods in mixed methods research include the need to validate the methodological approach particularly in disciplines that are dominated by other methodologies, often training to use particular methods, communicating the research purpose, design and findings, and also articulating appropriate data analysis strategies (Clark \& Ivankova, 2016; Creswell \& Plano Clark, 2017; Pauwels, 2007; Shannon-Baker \& Edwards, 2018). Research studies like Rule \& Harrell, (2010) utilized visual methods primarily, but analysed visual data using qualitative methods and the integration of visual data included transformation into quantitative data for further analysis and triangulation. For O'Connell (2013), visual methods were embedded in the qualitative research design and visual data was contextualized using other qualitative data. Here, there was integration of visual data that also included transformation into quantitative data and the construction of the case studies. The other exemplar is by Shannon-Baker \& Edwards, (2018) that uses visual methods as part of an arts-based critical visual research methodology. The commonalities identified in these studies using visual methods is that firstly, participant created visual data is used and also visual data is transformed to quantitative data so that both quantitative and qualitative strategies reinforce and legitimize visual methods.

\subsection{Realist Positivism vs Social Constructivism}

The visual approach has been conventionally grounded on a realist positivist approach that looks upon visual images and data as the objective reality and to be regarded as unbiased and unmediated representations of the social world (Ortega-Alcázar, 2012). Modern contemporary views challenge these assumptions and positivist epistemologies so there is currently a debate on the presumed objectivity and the unambiguity of visual data. Social constructivism 
takes into perspective the subjective presence of the person behind the camera who plays a crucial role in framing the image captures, the polysemic nature of visual representation and the idea that audiences are not passive consumers but also constructors of meanings and interpretations of the visual. Visual materials through the use of digital photography and videography are acknowledged to be subject to multiple interpretations and perspectives so hold no fixed or single meaning. Images and visual representations have the power construct specific visions of social class, race, and gender and can provide particular perspectives of the social world, thus having an important influence on audiences or those who consume these images.

\subsection{Analysis and Interpretation of Visual Materials}

The acknowledgement of the possible ambiguity of meaning and acknowledgment of the polysemic quality of visual representations has opened the field for the analysis of these images in various contexts including marketing materials, models, and communication to certain groups of audiences. The main methods of analysis of visual materials and data are i) content analysis ii) semiotic analysis iii) discourse analysis (Ortega-Alcázar, 2012). The approach of content analysis of visual data is often a clearly defined methodological process that seeks to produce valid and replicable findings. This approach may be based on counting the frequency in which a certain element or quality appears in a defined set of images. Content analysis would then serve to provide a descriptive account of the content of a given sample set of images rather than the interpretation of various possible meanings. This may help to identify trends through image data sets and certain software applications. NVIVo Ncapture for instance can work with large data sets on Facebook posts to provide this form of analysis that has a quantitative aspect in it.

The second method to the analysis of visual data is the use of semiotic analysis. This approach is grounded on the theory of Swiss linguist, Ferdinand de Saussure who proposed that the sound of speech and signs have no intrinsic meaning, but meanings are ascribed through linguistic signs that are made of the signifier and the signified. The relationships between the signifier and the signified are arbitrary. Poststructuralists challenge the concept by Saussure that once the signifier and signified are integrated to forms a sign, the sign has a fixed meaning. Poststructuralist theory and semiotics argue that meanings are not fixed but are continually being open to interpretation as signifiers are detachable from the things that are being signified. Barthes developed Saussure's theory to argue that there are two levels of signification, denotation and connotation. The first level is the literal (denotative) and at the second level, signs can have other attached meanings (connotative). 
The third form of interpretation is that of discourse analysis and stems from a critique of the realist approach to language. It claims that meaning is constituted within language and therefore language is constitutive of the social realm. Discourses are constructed from a series of related statements (both visual and textual) on a particular topic or theme and make up an authoritative language for speaking about the topic and shape the way a particular topic or issue is understood and interpreted. It does not attempt to read or analyse images but seeks to understand what the images or text claim is the 'truth'.

\subsection{Grounded Theory and Visual Analysis}

Ethnographic research is used to document events, objects and activities of interest. This has led to a collective analysis of participant-generated images rather than researcher generated digital documentation. The site or sites of data collection may be expanded by visual participatory methods or participant representation of activities and events in spaces and places that the researcher would normally not have access to (Hicks, 2018). Such visual methods may allow participants across linguistic, social and geographical divides to visually represent what may not always be visible or accessible to the researcher or audience outside the setting (Greyson et al., 2017). The use of visual methods expands grounded theoretical approaches by diversifying the data that the researcher has access to. While photographs and videography may not form a wholly objective representation of reality, participant generated images help to magnify and elaborate an understanding of the social enactment of activities, interactions and relationships through a detailed and multi-faceted perspective (Croghan et al., 2008). In allowing participants, a means to portray and represent what is of priority and importance to them rather than what is important to the researcher alone. Constructivist grounded theory transpires through the understanding that meaning is co-constructed between research participant and researcher rather than merely brought into existence through an objective and neutral observer (Charmaz, 2015).

\section{3}

\section{Description of the Research Scenario}

The research settings included various centres in Singapore and these were of three main types: privately owned, corporately owned and community-based early childhood centres. Although the study was based on an exploratorysequential mixed methods design, the methodology and some of the findings shared in the context of this paper will be mostly limited to those derived from 
visual research methods and would not discuss the quantitative findings. The initial method used with internet-based visual data aimed to obtain a visual account of how the curriculum was enacted in the different learning environments and centre types. The priorities and commonalities in the activities and curriculum programmes in these settings were also investigated through data generation and analysis using visual research methods that included: i) internet based visual data ii) participant generated data and iii) image or photoelicited data.

\subsection{Internet-based Visual Data}

The first stage of data generation involved social media data or essentially posts by a selection of centres. These centres were a representative sample using social media or Facebook posts over a period of 12 months. The posts that were selected fulfilled certain criteria and were images captured i) involving the children as active participants in the learning environment ii) involving both children and teachers and/or facilitators engaged in activity iii) involving children, teachers and parents involved in an event or participating in activity. It was essential to note that the learning environment was not always within the ECE centre setting itself but also constituted of the environments that the class was immersed while on field trips and excursions. The constantly transforming environment within the centre itself during various festivities and celebrations was also observed and captured in the posts over the period of time.

Each social media Facebook post consisted of a cluster of photographic images capture during a particular activity or event (Figure 1 and 2). In total, the sample demonstrated here were 72 such posts by five different representative early childhood education centres. Each of these main posts was coded via ground theory analysis and the distribution of frequency for each thematic code is represented in Table 1. As coding of the visual materials is often arbitrary and often subject to personal judgment, the images were also represented by text with short bulleted points based on the visual and caption or commentary that accompanied the image (See Figure 2). The visual image was there also represented in text and this was also coded into the various themes.

Based on the percentage distribution of the total frequency of 733, it showed that certain thematic codes (Figure 3) were well represented in these media posts with a relative heavier emphasis of 'Discovery of the World' domain from the national curriculum framework curriculum or the NEL framework (Nurturing Early Learners). Another inductive theme that was used was 'Integrated MI or Multiple Intelligences' which referred to activities that engaged more than one NEL domain or two or more of the eight Gardner's Intelligences (e.g. 


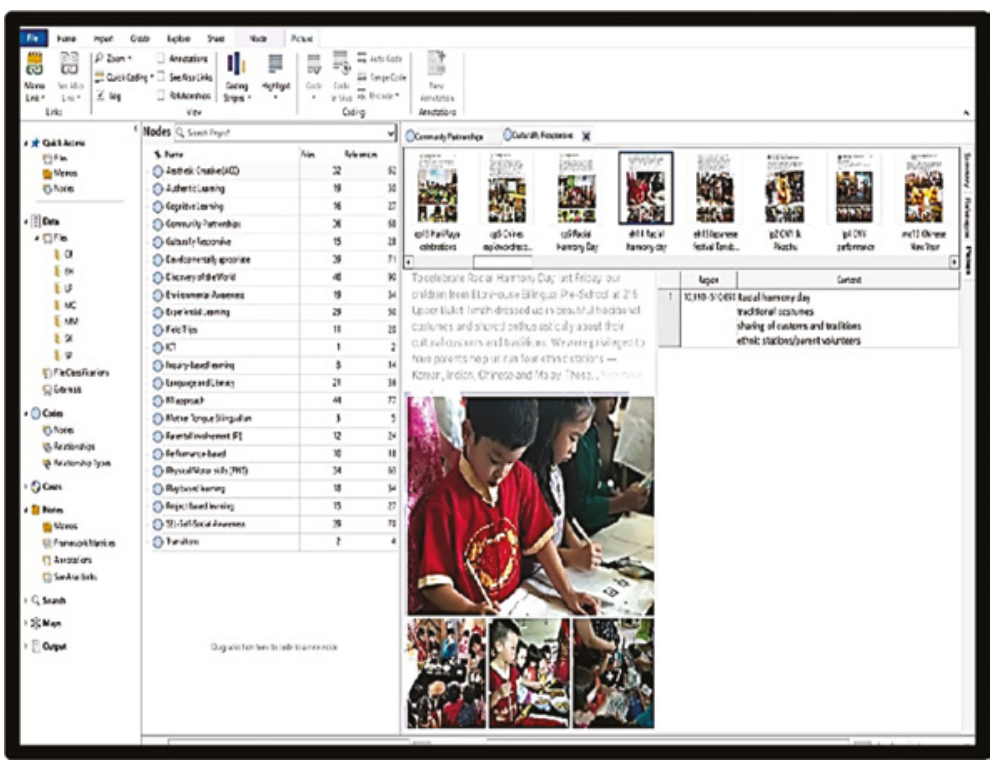

FIGURE 1 Thematic coding with NVIVo12 Pro

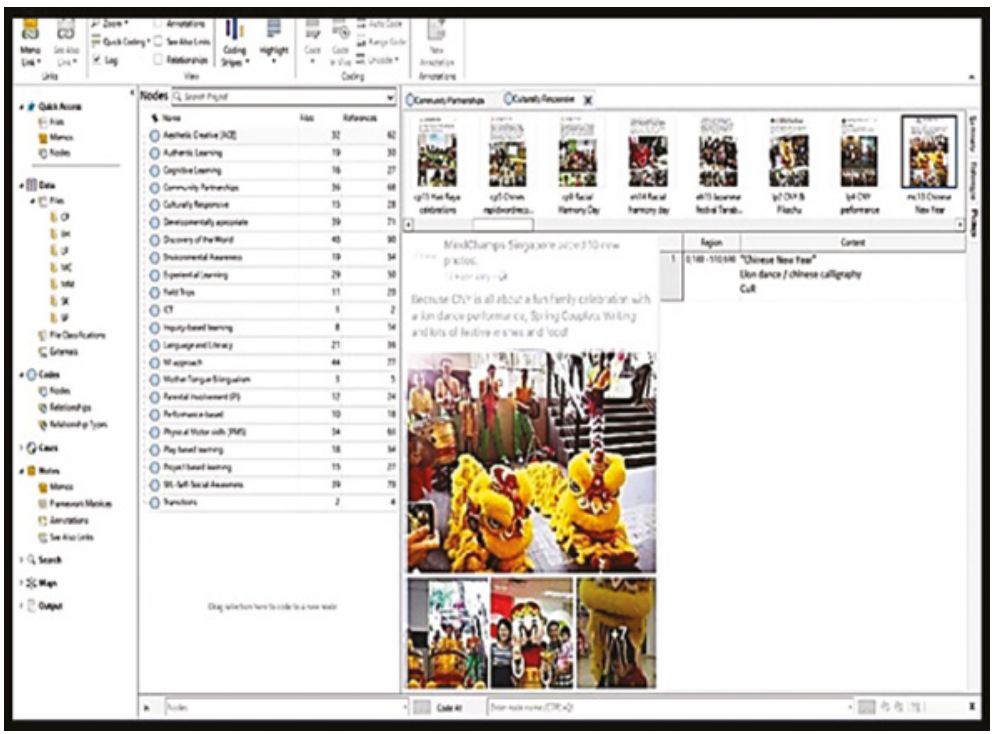

FIGURE 2 NVIVO image-pic view of selected code 
TABLE 1 Frequency and percentage distribution of thematic codes.

Thematic codes:

NEL Framework domains

- Discovery of the world (87) $11.9 \%$

- Self and Social Awareness (65) 8.9\%

- Aesthetic and Creative (6o) 8.2\%

- Physical Motor Skills (6o) $8.2 \%$

- Cognitive (Numeracy) (26) 3.5\%

- Language and Literacy (19) $2.6 \%$

Integrated $M I(73) 10.0 \%$

Developmentally appropriate (66) $9.0 \%$

Community Partnerships (63) $8.6 \%$

Experiential learning (49) $6.7 \%$

Environmental Awareness (33) 4.5\%

Culturally Responsive (28) 3.8\%

Project-based learning (27) 3.7\%

Authentic learning (27) $3.7 \%$

Parental involvement (22) $3.0 \%$

Performance based ( 18$) 2.5 \%$

Transitions (4) $0.5 \%$

Mother tongue / bilingualism (4) $0.5 \%$

ICT $(2) 0.3 \%$

logical-mathematical, verbal-linguistic, naturalistic, visual-spatial, intrapersonal, interpersonal, musical, and kinesthetic). The 'Cognitive' domain of the NEL framework was supplanted by 'Numeracy skills' as a great percentage of activities engaged the cognitive skillset but this was not easily specifically identified.

Many of the posts featured in these ECE social media postings featured activities that were specific to different levels such as sessions that encouraged hand-eye coordination and aesthetic expression for 3-4-year olds (Nursery 1 ) or more cognitively advanced activities such as projects that required higher level critical thinking and reflection with the $5^{-6}$-year olds (Kindergarten 1 ). Such activities emphasized the developmental appropriateness of the skills subsets required to participate actively in them. Some of these posts involved mixed age groups particularly in festive celebrations and assembly activities, 


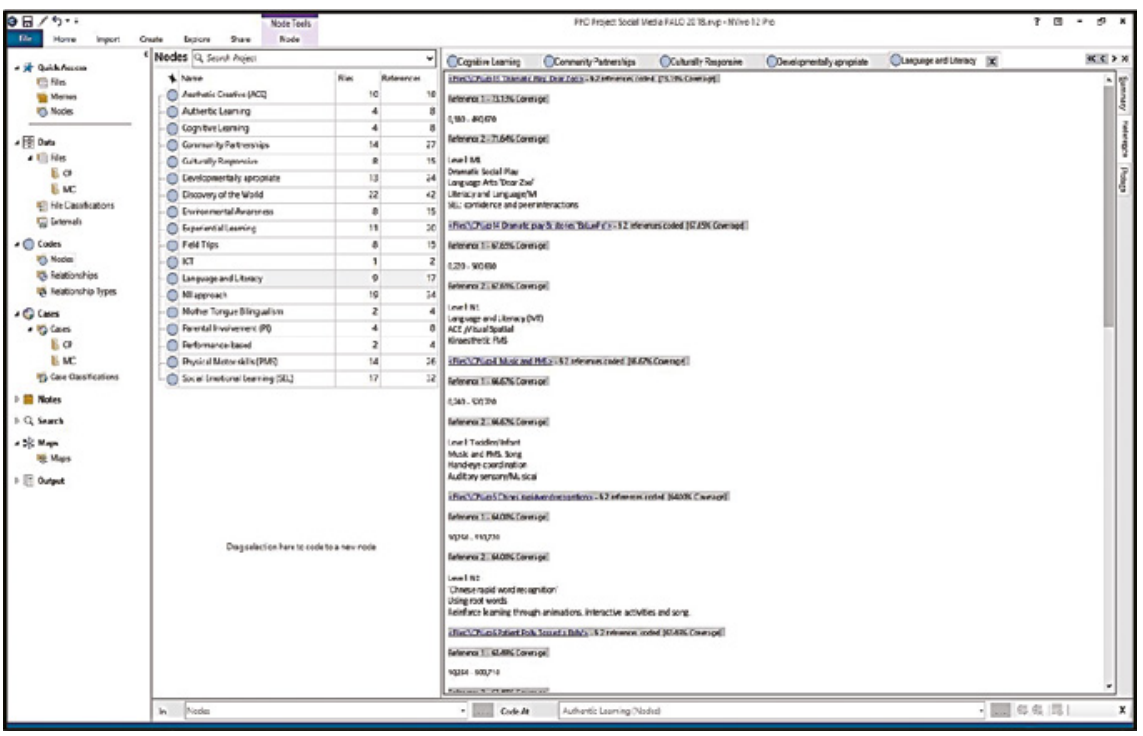

FIGURE 3 NVIVO Reference view of selected code

these allowed the various age groups and levels to participate in them. Of the 733 frequency counts of coding, 63 counts featured community partnerships and involvement in some form of another. These community partnership activities allowed the children to experience and immerse in different learning environments including the neighborhood and community surroundings such as the fire station, community gardens, hydroponic vegetable, goat and even frog farms around the island. Experiential learning in the form of interactive, hands-on experiences is involving the senses and sometimes situated in reallife contexts as in authentic learning (Reed \& Canning, 2013). In learning science and mathematical concepts, the interaction with material with resultant play and creativity are noted as forms of experiential learning. Other codes that were used included activities that promoted environmental awareness (33), culturally responsive curriculum (28) and project-based learning (27).

The visual data in these thematic codes include activities and events such as gardening, outdoor field trips for environmental awareness, celebration of various festivals, racial harmony day that was an aspect of a culturally responsive curriculum. It was noted that project-based learning usually involved those four years and above as these required higher order thinking and 


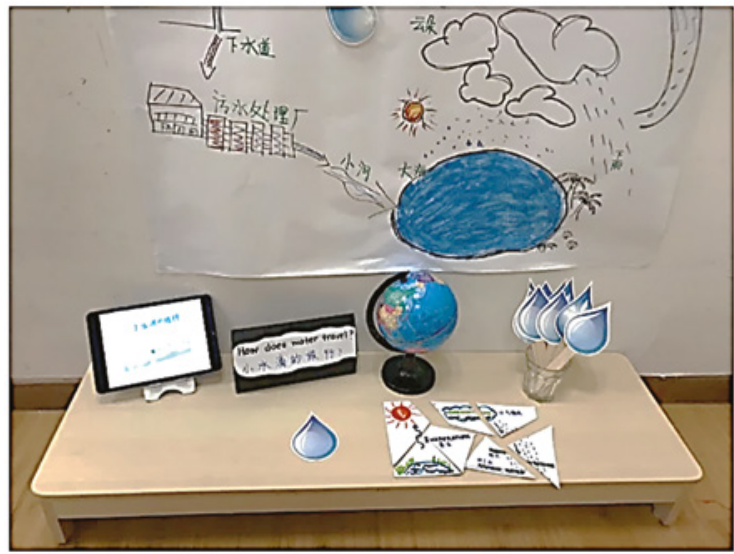

A story about water is read

Follow the story line which shows how water travels

Count water droplets, counting from 1-10 and counting on

Complete a simple puzzle on the water cycle with a friend

FIGURE 4 Planning learning spaces

problem-solving activities. Certain thematic codes were relatively less represented in these social media posts such as mother tongue activities although they may form a core aspect of the curriculum perhaps due to the nature of these activities which does not lend itself readily to visual representation in such media.

\subsection{Researcher and Participant-generated Visual Material}

Participant-generated visual data may use different forms of images including photographs, video clips, artefacts, drawings and work samples, together with other forms of visual representations. In this study, teacher participants were asked to select at least three artefacts or examples of work that their students had worked or made during class activities. This appeared to be selective emphasis of the products rather than on the processes of the curriculum. There was also examples of photographs and short video clips that demonstrated the processes of the curriculum and what was important or of priority to the teacher participants themselves. It was found to be very effective in communicating the processes in the curriculum through photo documentation series with explanatory texts accompanying these.

Photographs that are generated research contexts are often a product of the network of relations between the participant, the researcher and the audience/s and the debate ensues that there should be not one meaning ascribed, but the possibility of multiple interpretations and meanings that could evolve over time or remain relative unchanging. The meaning could also be a co-construction between participant and researcher (Drew \& Guillemin, 2014). 


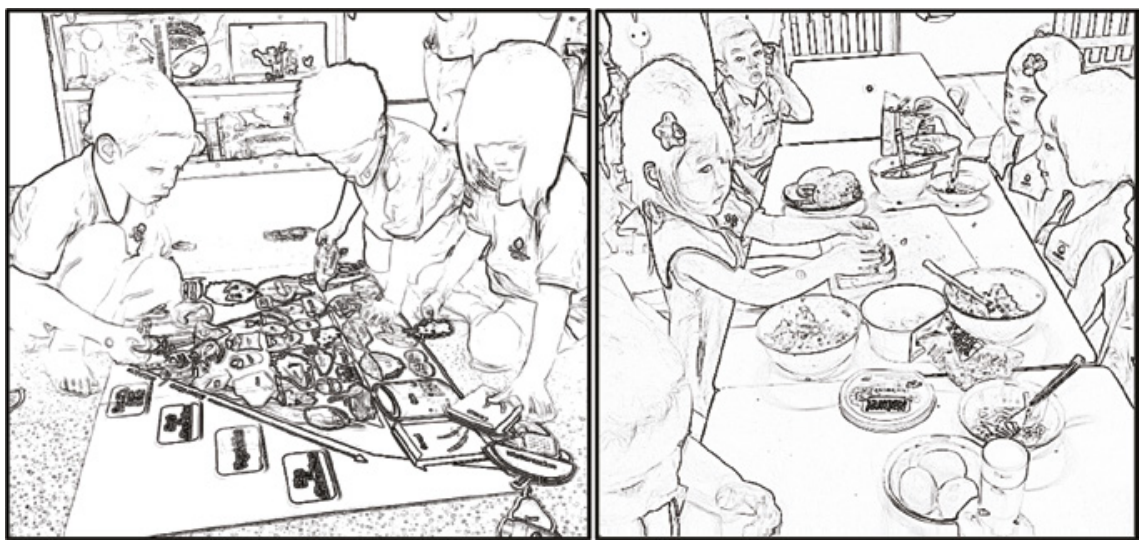

FIGURE 5 Learning about the food pyramid and a balanced meal

Interviewer:

Teacher Participant

Interviewer:

Teacher Participant

Interviewer:

Teacher Participant
These photos show that the class is quite interested in what looks like a puzzle.

Yes. These activities were part of my weekly plan on the theme of 'My Body'. I took the opportunity to let them explore and learn something about the food pyramid followed by a healthy sandwich making activity for their teatime snack.

What are they actually doing with the food pyramid?

I introduced the food pyramid that in this case is a large board display with the different levels labelled. The food items are in the form of picture magnets. Initially, we had a bit of a discussion with questions that provoked their thinking. For example: Each level consists of a food group so questions like 'what group do you think broccoli or beans should place in?' There were a few calculated guesses as we went along. After this, I allowed 2 or 3 of them to take turns sort the different foods into the appropriate food groups. This was one of their learning corner activities....

That sounds good and they seem quite engaged in grouping and categorizing the different items that is part of their cognitive or logico-mathematical skill subset.

The group activity also gives them the opportunity to interact and communicate with their peers that is an important skill as well.... The healthy sandwich making 
session gave them the opportunity to practice handling utensils and their finer motor skills and at same time learn some self-help skills that nurtures their selfesteem and independence in daily activities.

In some instances, the photographs themselves present a visual narrative even without further explanation from the individual participant or interpretation from the researcher. Although not shared by all researchers, Sarah Pink (2007) is particular about practices that subordinate the visual image to the written word in research. Drew \& Guillemin (2014) assert that a robust visual analytic process incorporates both the participant and researcher voices, while relating these various layers of perspective and statements made so as to demonstrate the emerging analytical narrative that may become emphasized or diminished based on the overall research direction and objectives. They point out three stages to interpretative visual analysis and meaning making when using participant-generated visual material although not all analysis passes through all three stages. The first stage is that of meaning making through the engagement of the participant and image production. This stage of analysis engages mainly with the stories, experiences and representations that participants wish the researchers to know about through the participant's reflections on the visual material generated and the participant guides the way they feel the visual material should be interpreted. The second stage of the interpretative process involves a closer examination of the visual materials and that of the participant's explanations. The researcher's reflections on these facilitates the forming of themes and the interconnections between these themes, the context in which these visual materials were generated, together with other details will provide further interpretation of the participant's reflections. This could also include the participant's interview responses on further probing and inquiry into the participant's interpretation or processes. Stage process refers to meaning making through re-contextualization within the theoretical and conceptual frameworks to define and identify the emerging analytic patterns. This stage allows a more final and defined robust analytic explanation.

\subsection{Visual/Photo-elicited Data}

The visual research method used here refers to the use of images, photographs, drawings or other work samples or artefacts from the teacher participants themselves or from the students in their class (Ferguson, 2013). In some instances, participants were specifically given the equipment to capture the images that were used at a later stage for stimulating discussion and reflection 
(Croghan et al., 2008; Dunne et al., 2017). Both researcher and participantgenerated visual data was also often used in a photo-elicited semi-structured interview setting. However, not just direct participant-generated images but also work samples and artefacts from their classrooms, particularly when direct field observations were not always possible in elucidating the processes of creation and generation of the artefacts. Banks, (2007) elaborates on photoelicitation by itself and refers to it as involving photographs to invoke memories, comments and discussion during the course of semi-structured interview. The visual material may be participant-generated as mentioned in the earlier section, directly or indirectly or it may be researcher generated photographs or digital video clips. The framing of the visuals may demonstrate certain examples of inter-relationship and social interactions and provide a detail of the cultural context of the activity or event represented. These may provide the basis for discussion and elaboration of the abstraction, trigger details, and focus during the process.

Perhaps what is missing in this context are the children's direct voices and their own meaning-making through their work. As the dialogue with the teacher participants sometimes, takes place a period after the creation of their artwork and there was insufficient opportunity to take the time to dialogue
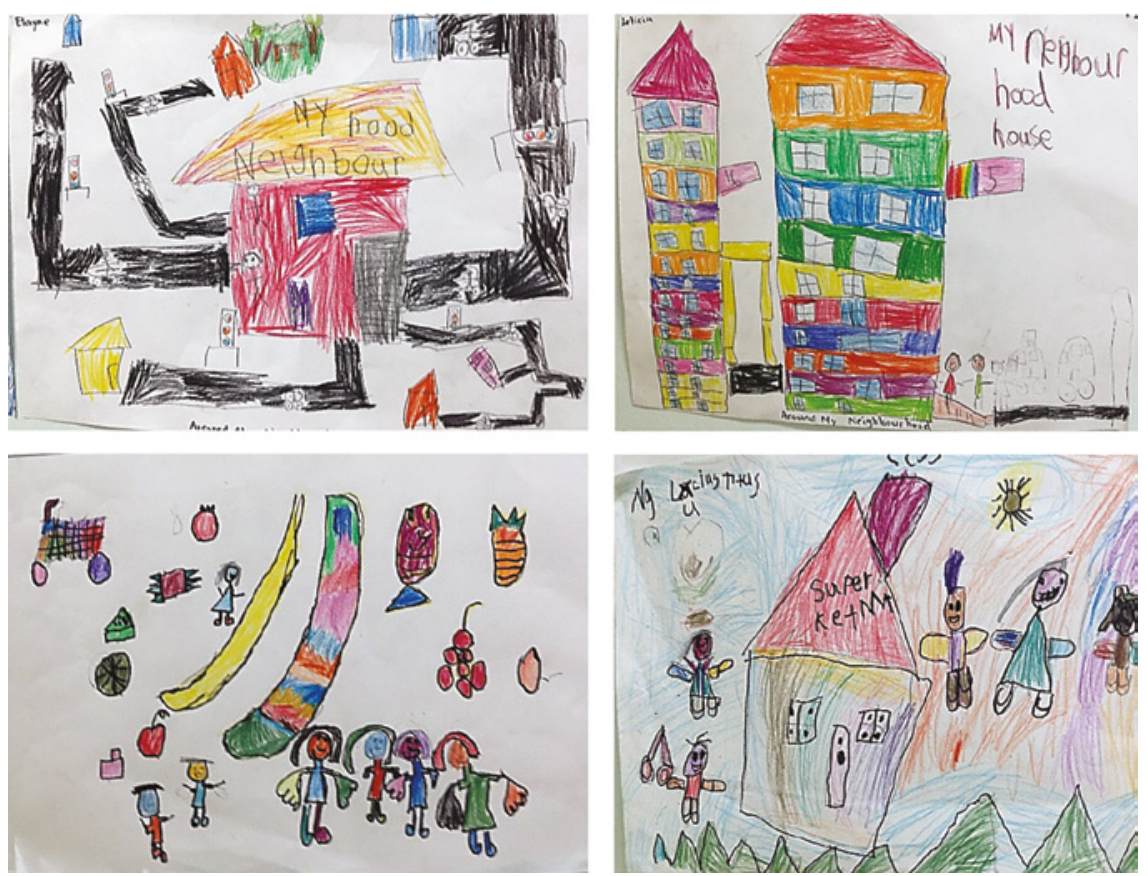

FIGURE 6 The supermarket in the neighbourhood 
Interviewer

Teacher Participant We were working on a thematic unit: Our Neighbourhood and Community so there were a number of projects that the class was working on. We had a walk-about outing to the nearby supermarket as well and the children each had a $\$ 5$ budget to buy something healthy. They loved the fruit section of the supermarket and the variety of fruits available. When we got back from the walk, the children discussed about what they saw and learnt during the outing. They then wrote in their journals. There was no lack of materials, colours and art materials for them to freely express themselves.

Interviewer Teacher Participant So, there is quite a lot of interconnection between one activity and another during the thematic unit? Yes, as thematic unit allow us to work on several different aspects of the theme. At this level, a 5-year-old are much more capable than a 3-year-old, has more prosocial skills and is better able to work collaboratively with his peers. They are also beginning to spell and write with some help. Over a course of a few weeks, the class also worked on a 3-dimensional model project using recycled materials such as cereal boxes, egg cartons and so on.

directly with the children but rather to learn about the process through the teacher participants' perspective at this stage. The meaning-making process here considers mainly the interpretation of the teacher and the researcher. The fact is that images should be acknowledged to be multi-vocal, having the ability to 'speak' to different audiences in a variety of contexts (Banks, 2011; YamadaRice, 2014).

In current times, digital media has reached into our mundane everyday existences, most obviously through the cell phone and modern-day gadgets, social media and these digital artefacts have engendered new forms of ethnographic enquiry. One of these includes what might be termed as the cultural politics of media and examines cultural identities, representations and imaginaries (Coleman, 2010). Fleer \& Ridgway, (2014) outline and frame visual narrative 
data based on cultural-historical theory. Cultural historical theory acknowledges that the characteristics of individuals engaged in activities and interactions within a certain cultural setting can evolve and transform over a period of history. This can enable the researcher a better understanding on why certain practices and needs are defined as they are in a specific context and that different perspectives and priorities are taken in different cultures and times (Fleer \& Ridgway, 2014; Gutiérrez, Engeström, \& Sannino, 2016).

Though field observation, particularly in Reggio-Emilia inspired centres and where children are given free reign of their imagination through encouragement and access to materials, it has been observed that the young can use the graphic and expressive languages of drawing, painting, collage and construction to record their ideas, observations, reflections, memories to further explore their understanding. Embedded in these activities are the processes of reconstructing and building on earlier knowledge so as to externalize their thoughts and what is learnt, to share their worlds with their peers and others (Malaguzzi, 1994; Wright, 2007). The approach using 'art as epistemology' (Gallas, 1991) so that art experiences in the classroom can have both communicative and expressive goals, and the concept of art as a symbolic language is the subject of much debate. This highlights the potential of teachers facilitating children to develop the capacity in the 'hundred language' that is accessible to them so as to master the range of instruments and symbols (Vygotsky \& Luria, 1978) that form the visual culture and an expressive language used in the curriculum. The potential for research based on visual methodologies is thus boundless.

\section{Acknowledgements}

I would like to thank NIEC, National Institute of Early Childhood Development, Singapore and the following teacher participant contributors Chandra Rai, Shauna Chen and Kavita Mogan.

\section{References}

Banks, M. (2007). Visual methods and field research. In Using Visual Data in Qualitative Research, 58-91. SAGE. https://doi.org/10.4135/9781526445933.n4.

Banks, M. (2011). Presenting visual research. In Using Visual Data in Qualitative Research, 105-128. SAGE. https://doi.org/ 10.4135/9781526445933.n5. 
Bronfenbrenner, U., \& Morris, P. A. (2006). The Bio ecological Model of Human Development. In Handbook of child psychology: Vol. 1. Theoretical models of human development. John Wiley.

Charmaz, K. (2015). Grounded Theory: Methodology and Theory Construction. International Encyclopaedia of the Social \& Behavioural Sciences: Second Edition (Second Ed, Vol. 1o). Elsevier.

Clark, V. L. P., \& Ivankova, N. V. (2016). How to Expand the use of Mixed Methods Research?: Intersecting Mixed Methods with Other Approaches. In Mixed Methods Research: A Guide to the Field, 135-160.

Coleman, E. G. (2010). Ethnographic Approaches to Digital Media. ssRN. https://doi .org/10.1146/annurev.anthro.012809.104945.

Creswell, J., \& Plano Clark, V. (2017). Designing and conducting mixed methods. (Third, Ed.). SAGE.

Croghan, R., Griffin, C., Hunter, J., \& Phoenix, A. (2008). Young people's constructions of self: Notes on the use and analysis of the photo-elicitation methods. International Journal of Social Research Methodology, 11(4), 345-356. https://doi.org/10 $.1080 / 13645570701605707$.

Croghan, R., Griffin, C., Hunter, J., \& Phoenix, A. (2008). Young people's constructions of self: Notes on the use and analysis of the photo-elicitation methods. International Journal of Social Research Methodology, 11(4), 345-356. https://doi.org/10 $.1080 / 13645570701605707$.

Danko-Mcghee, K. (2009). The Environment as Third Teacher: Pre-service Teacher's Aesthetic Transformation of an Art Learning Environment for Young Children in a Museum Setting. International Art in Early Childhood Research Journal, 1(1), 1-17.

Denzin, N. K. (2012). Triangulation 2.0*. Journal of Mixed Methods Research, 6(2), 8088. https://doi.org/10.1177/1558689812437186.

Drew, S., \& Guillemin, M. (2014). From photographs to findings: visual meaning-making and interpretive engagement in the analysis of participant-generated images. Visual Studies, 29(1), 54-67. https://doi.org/10.1080/1472586X.2014.862994.

Dunne, L., Hallett, F., Kay, V., \& Woodhouse, C. (2017). Visualizing Inclusion: Employing a Photo-Elicitation Methodology to Explore Views of Inclusive Education. https://doi .org/10.4135/9781473995215.

Ferguson, T. S. (2013). Social Research Methods (3rd Edt). Oxford University Press.

Fleer, M., \& Ridgway, A. (2014). Visual methodologies and digital tools for researching with young children: Transforming visuality. Visual Methodologies and Digital Tools for Researching with Young Children:Transforming Visuality. https://doi.org/10.1007/ 978-3-319-01469-2.

Flick, U. (2018). Doing triangulation and mixed methods. SAGE. 
Gallas, K. (1991). Arts as Epistemology: Enabling Children to Know What They Know. Harvard Educational Review, 61(1), 40-51. https://doi.org/10.17763/haer.61.1 .l7518t5kho6710n4.

Greyson, D., O'Brien, H., \& Shoveller, J. (2017). Information world mapping: A participatory arts-based elicitation method for information behaviour interviews. Library and Information Science Research, 39(2), 149-157. https://doi.org/10.1016/j .lisr.2017.03.003.

Gutiérrez, K. D., Engeström, Y., \& Sannino, A. (2016). Expanding Educational Research and Interventionist Methodologies. Cognition and Instruction, 34(3), 275-284. https://doi.org/10.1080/07370008.2016.1183347.

Hicks, A. (2018). Developing the methodological toolbox for information literacy research: Grounded theory and visual research methods. Library and Information Science Research, 40(3-4), 194-200. https://doi.org/10.1016/j.lisr.2018.09.001.

Malaguzzi, L. (1994). Your image of the child: Where teaching begins. Child Care Information Exchange, $3(800), 5^{2-56 .}$

Metcalfe, A. S. (2016). Educational research and the sight of inquiry: Visual methodologies before visual methods. Research in Education, 96(1), 78-86. https://doi .org/10.1177/0034523716664577.

Miller, D. (2011). Tales from Facebook. Polity Press.

Moss, J. (2013). Visual research methods in education: In between difference and indifference. International Journal of School Disaffection (Institute of Education Press), $10(2), 63-78$.

O'Connell, R. (2013). The use of visual methods with children in a mixed methods study of family food practices. International Journal of Social Research Methodology, 16(1), 31-46. https://doi.org/10.108o/13645579.2011.647517.

Ortega-Alcázar, I. (2012). Visual research methods. International Encyclopedia of Housing and Home, (pp. 249-254). https://doi.org/10.1016/B978-0-08-047163-1.00693-7.

Pauwels, L. (2007). An Integrated Conceptual Framework for Visual Social Science Research. In The Sage Handbook of Visual Research Methods.

Pink, S. l. (2007). "Visual Methods." Qualitative Research Practice. 361-376. https://doi .org/10.1109/61.568259.

Pink, Sarah (Ed.). (2012). Visual ethics in a contemporary landscape. In Advances in Visual Methodology. SAGE.

Pink, Sarah. (2014). Advances in Visual Methodology. Advances in Visual Methodology. https://doi.org/10.4135/9781446250921.

Prosser, J. (2007). Visual methods and the visual culture of schools. Visual Studies, 22(1), 13-30. https://doi.org/10.1080/14725860601167143.

Reed, M., \& Canning, N. (2013). Reflective practice in the early years. International Journal of Early Years Education, 21(1), 1-202. https://doi.org/10.4135/9781446288924. 
Rule, A. C., \& Harrell, M. H. (2010). Symbolic Drawings Reveal Changes in Preservice Teacher Mathematics Attitudes After a Mathematics Methods Course. School Science and Mathematics, 106(6), 241-258. https://doi.org/10.1111/j.1949-8594.2006.tb 17913.x.

Shannon-Baker, P., \& Edwards, C. (2018). The Affordances and Challenges to Incorporating Visual Methods in Mixed Methods Research. American Behavioural Scientist, 62(7), 935-955. https://doi.org/10.1177/0002764218772671.

Strong-Wilson, A. T., Ellis, J., Theory, S., Practice, I., winter, R. E., Strong-Wilson, T., \& Environment, E. (2016). As Third Teacher Children and Place: Reggio. Theory into Practice, 46(1), 40-47.

Tudge, J. R. H., Merçon-Vargas, E. A., Liang, Y., \& Payir, A. (2017). The importance of Urie Bronfenbrenner's bio ecological theory for early childhood education. Theories of Early Childhood Education: Developmental, Behaviourist, and Critical, (pp. 45-57). https//.doi.org/10.4324/9781315641560.

Vygotsky, L., \& Luria, A. (1978). Tool and Symbol in Child Development. In M. Cole \& V. John-Steiner (Eds.), Mind in Society: The Development of Higher Psychological Processes, (pp. 99-174). Harvard University Press.

Wright, S. (2007). Young children's meaning-making through drawing and 'telling': Analogies to filmic textual features, Australia Journal of Early Childhood, 32(4), 3749. https://repository.nie.edu.sg/handle/10497/16986.

Yamada-Rice, D. (2014). Using Multimodal Social Semiotic Theory and Visual Methods to Consider Young Children's Interaction with and Comprehension of Images. Using Multimodal Social Semiotic Theory and Visual Methods to Consider Young Children's Interaction with and Comprehension of Images. https://doi.org/10.4135/97814 4627305013509947. 